\title{
Editorial
}

\section{Umweltfolgen und Arbeitsbedingungen als Akzeptanzprobleme}

\author{
Prof. Dr. Marco Althaus \\ Technische Hochschule Wildau
}

Mit der dritten Ausgabe von JEMPAS gehen wir in den zweiten Jahrgang dieser Zeitschrift. Wir freuen uns über die Aufmerksamkeit und den Zuspruch für unsere Themen. Für die studentischen Autoren ist das Wagnis, sich erst dem Peer Review und dann der Online-Veröffentlichung zu stellen, nie eine Kleinigkeit und schon gar nicht Routine. Das gilt natürlich auch für unsere studentischen Gutachter des ersten JEMPAS-Jahres.

Die akademischen Aufsätze dieser Ausgabe sind in zwei Studiengängen der Technischen Hochschule Wildau entstanden. Aus dem Master-Studiengang Europäisches Management stammen drei, aus dem Programm Master of Aviation Management zwei.

Der Kurs „European Economic Policy“ beschäftigte sich im Sommersemester 2014 unter anderem intensiv mit der Eurozone, der Schuldenkrise und den PIIGS-Staaten. Marcus Pahl recherchierte, wie es Griechenland gelang, auf den internationalen Finanzmärkten neue Gläubiger und Abnehmer für seine Staatsanleihen zu gewinnen. Pahl kommt dabei zu überraschenden Ergebnissen - und einer differenzierten Bewertung.

Im Kurs „Corporate Social Responsibility“ im Wintersemester 2013-14 arbeiteten studentische Teams an CSR-Problemen unterschiedlicher Branchen. Mikhail Aridov, Vanessa Becker, Svenja Golombek, Lisa Hamann, Jiyue Liu, Kaya Luschnat, Stephanie Niemuth, Paulina Smolarz und Anne Werner waren dabei. In ihren Teamprojekten ging es um Markenkaffee und Markenbekleidung: Konsumartikel, die alle Studierenden kennen. Der persönliche Bezug als Verbraucher erleichtert und erschwert den wissenschaftlichen Zugang zum Thema gleichermaßen. Denn sehr schnell wird sichtbar, dass nicht nur die Unternehmen Verantwortung tragen, sondern ebenso die Kunden darunter Studenten des Kurses.

Bei beiden Projekten war charakteristisch, dass die Produkte zwar hier gekauft, aber am anderen Ende der Welt hergestellt werden. Was die Unternehmen als gesellschaftliche Verantwortung tragen müssen, wird vor allem in den Verbraucherländern thematisiert, aber die Probleme und Lösungen sind oftmals am Anfang der globalisierten Wertschöpfungskette zu finden.

Weil die Probleme beim Konsum erst einmal sehr weit weg scheinen, sind sie zunächst leicht zu ignorieren. Umso schockierender ist es oft, wenn Medienberichte und kritische Aufklärungskampagnen zeigen, wer welchen Preis für den Konsum zu zahlen hat. Besonders deutlich wurde dies 2013 beim Einsturz des Rana-Plaza-Fabrikkomplexes in Bangladesh. Dass die Arbeitsbedingungen für die Textilarbeiter dort schlecht sind, ist allgemein bekannt; wie sehr es aber um Gefahren für Leib und Leben geht, erkannten viele Verbraucher erst zu diesem Zeit- punkt. Innerhalb sehr kurzer Zeit reagierten führende Unternehmen Europas mit einem überraschend klaren Schritt, der Unterzeichnung des Bangladesh Accord on Fire and Building Safety. Dieses im Grunde sehr positive Ereignis hat, wie die studentische Arbeitsgruppe herausarbeitete, allerdings keinen Anspruch auf kritiklosen Enthusiasmus. Die nüchterne Analyse belegt, wie komplex das Feld der Arbeitsbedingungen in den Niedriglohnländern ist. Internationale Bekleidungsunternehmen, die die CSR ernst nehmen, haben einen sehr mühevollen Weg vor sich.

Deutschlands prestigereiche Flughäfen wachsen, weil der internationale Luftverkehr wächst. Frankfurt und München profitieren als Drehkreuze in einem sehr globalen Geschäft. Ihre Wachstumsstrategien entwickeln sich im globalen Wettbewerb. Aber die örtlichen Anwohner und Anrainer können sich über den Erfolg der für ihre Region so wichtigen Arbeitgeber und Steuerzahler nicht immer freuen. Sie tragen die Last des Ausbaus für diese internationale Infrastruktur. Für sie ist die gesellschaftliche Verantwortung der Airport-Unternehmen ein lokales und regionales Thema - ob ökologisch geprägt oder anders definiert.

Tim Wiebusch und Franziska Ruh haben sich der Herausforderungen angenommen, die deutsche Flughäfen bei der Expansion bewältigen müssen. Ihre Artikel sind Produkte des Kurses „Aviation \& Society“ im Wintersemester 2013-14 im Studiengang Master of Aviation Management am Wildau Institute of Technology (WIT), dem An-Institut der Hochschule für Weiterbildungsstudiengänge, die sich an Berufstätige richten. Die Autoren sind in Unternehmen der Luftfahrtbranche tätig. Sie arbeiten nicht für die untersuchten Flughäfen, aber fühlen sich dort natürlich zu Hause. Mit einem durch die Branche geschärften Blick haben sie genau beobachtet und ausgewertet, wie sich die AirportUnternehmen den gesellschaftlichen Anforderungen stellen.

Die letztgenannten vier Beiträge zur gesellschaftlichen Verantwortung haben gemeinsam, dass es am Ende um Legitimations- und Akzeptanzprobleme von Industrie- und Dienstleistungsunternehmen geht. Zwischen Infrastruktur-Großprojekten und dem Handel mit Konsumartikeln liegen einerseits Welten, andererseits aber ist der öffentliche Druck auf das Management, die Geschäftspolitik im Geflecht der Stakeholderkommunikation nach Nicht-Markt-Aspekten auszurichten, doch wieder ähnlich.

In beiden Sektoren haben wir uns darin gewöhnt, dass die Unternehmen offenen Dialog verfolgen, um die Akzeptanz abzusichern. Oft genug lassen sich aber die Kerndilemmata nicht so einfach wegdialogisieren und mühelose Kompromisse finden, die die Interessengegensätze ausgleichen und endgültig befrieden. Im Gegenteil: Das Management muss sich mit der Realität dau- 
erhafter Konflikte abfinden. Um Akzeptanz ist immer wieder neu $\mathrm{zu}$ werben und zu kämpfen. Das gilt für heimische, regionale Themen ebenso wie für die internationalen, globalen.

\section{sos}

In der Rubrik „Praxis“ geht es diesmal nach China. Die etablierten Beziehungen zwischen der TH Wildau und chinesischen Hochschulen ermöglichen seit einigen Jahren ein stabil verankertes Austauschprogramm. Im Bachelor-Studiengang Europäisches Management finden sich stets zahlreiche Bewerber, die sich unbedingt ein eigenes Bild vom Drachen machen wollen - mit Sprach- und Fachkursen, Exkursionen und Praktika.

Zwar zielt der Studiengang Europäisches Management vorrangig darauf, Nachwuchskräfte für den großen EU-Binnenmarkt vorzubereiten. Doch Europas Märkte enden nicht an Europas Küsten. Viele „Europa“-Studierende zieht es darum für ein Auslandssemester nicht nach Portugal oder Polen, Frankreich oder Finnland - sondern in vielversprechende Schwellenländer, ganz besonders in den Fernen Osten.

$\mathrm{Ob}$ es daran liegt, dass Reisen innerhalb Europas dank EasyJet \& Co. schon ,zu normal“ geworden sind? Oder dass ein karrierebewusster Lebenslauf für Wirtschaftswissenschaftler ohne Station in Asien nicht ausgereizt wirkt? Liegt es daran, dass Chinas alte Kultur gerade im Kontrast zur rasenden Moderne Chinas besonders attraktiv glänzt und funkelt? Oder daran, dass Chinas „roter Kapitalismus“ als Wirtschaftsmodell des 21. Jahrhunderts gilt, das Europa und Amerika als ganz alte Welt hinter sich lässt?

Vielleicht von allem ein bisschen. Neugier und Abenteuerlust haben viele Quellen. Nur wenige fühlen sich abgeschreckt durch politische Bauchschmerzen: Die jüngsten Protestwellen in Hongkong, die Missachtung von Bürger- und Menschenrechten, EinParteien-Diktatur, militärisches Säbelrasseln, soziale Verwerfun- gen und Umweltdesaster wecken bei Studierenden zwar Fragen, aber trüben das faszinierende Bild Chinas eher wenig. Die meisten hoffen, dass sich die Probleme mit fortschreitender Entwicklung lösen werden und dass sich mit der unumkehrbaren Öffnung des Landes Reformen leichter durchsetzen lasssen. Bis dahin ist China eben ,anders“.

Übelnehmen kann man die milde Haltung kaum. Sie drückt den Respekt vor der anderen Kultur ebenso aus wie die Vorsicht vor dem vorschnellen Urteil und die Bereitschaft, zwischen Staat und Menschen zu unterscheiden. Das ist auch gut so.

Der Pragmatismus spiegelt schließlich die Linie der deutschen und europäischen Außenpolitik wider. Unsere Wirtschaftsunternehmen tun ohnehin alles, um im Markt und bei der Errichtung des „chinesischen Traums“ (von dem Staats- und Parteichef Xi Jinping so oft spricht) dabei sein zu dürfen.

China ist, alles in allem, bei Studenten populär. Auf der Wunschliste für einen Auslandsaufenthalt steht China immer ganz oben. Und warum nicht? Wer sich traut und sich auch durch umfangreiche Vorbereitungen und Kulturschockerwartungen nicht abschütteln lässt, lernt eine Menge dazu.

So war es auch für Bianca Schwarzenburg, Bachelor-Studentin im Programm Europäisches Management. Schon mit vielen Stempeln im Reisepass ausgestattet, schildert sie in ihrem Reisebericht die Alltagserfahrungen, auf die die hiesigen Lehrveranstaltungen im Interkulturellen Management bestenfalls ansatzweise vorbereiten können, und die ersten Versuche in der chinesischen Sprache. Sie erläutert darüber hinaus, was sie aus dem Seminarprogramm an der Gasthochschule mitgenommen hat. Ihr ging es schließlich auch darum, das Wirtschaftssystem Chinas und des asiatischen Raums kennenzulernen. Ihr Essay ist eine muntere und erhellende Auseinandersetzung mit einem Land, das auch Europas Zukunft prägen wird.

\section{Impressum - Imprint}

\section{Journal of European Management \& Public Affairs (JEMPAS) \\ A Student Research Journal Published in English and German at Technical University of Applied Sciences Wildau, Germany}

\section{ISSN 2199-1618}

Head editor / Herausgeber, verantwortlicher Redaktionsleiter (V.i.S.d.P.)

Prof. Dr. Marco Althaus, Professor of Social Sciences / Professor für Sozialwissenschaften, Department of Business, Computing and Law / Fachbereich Wirtschaft, Informatik und Recht, Technische Hochschule Wildau [FH], Bahnhofstraße 1, 15745 Wildau, Germany E-Mail:marco.althaus@th-wildau.de

Editorial team and reviewers of this issue / Redaktion und Gutachter dieser Ausgabe

Dana Beisembiyeva / Stephen Bwete / Aurelia Dieterle / Huy Dinh / Anja Gocha / Stefan Mirsch / Marcus Pahl / Kseniia Romashova / Stephan Sienicki

JEMPAS is sponsored by and associated with Technical University of Applied Sciences Wildau, Germany. General in formation about this journal, as well as editorial policies including the student peer review process, rubric explanations, author guidelines and copyright notices are available at the journal's website www.jempas.th-wildau.de.

(C) 2014 by the authors.

JEMPAS is an electronic open access journal on a platform using Open Journal Systems 2.3.6.0, which is open source journal management and publishing software developed, supported, and freely distributed by the Public Knowledge Project under the GNU General Public License. 